\title{
Article \\ Flow-Particle Coupling in a Channel Flow Laden with Elongated Particles: The Role of Aspect Ratio
}

\author{
Alessandro Capone*, Fabio Di Felice (D) and Francisco Alves Pereira (D) \\ CNR-Institute of Marine Engineering, Via di Vallerano 139, 00128 Rome, Italy; fabio.difelice@cnr.it (F.D.F.); \\ francisco.alvespereira@cnr.it (F.A.P.) \\ * Correspondence: alessandro.capone@inm.cnr.it
}

Citation: Capone, A.; Di Felice, F.; Alves Pereira, F. Flow-Particle Coupling in a Channel Flow Laden with Elongated Particles: The Role of Aspect Ratio. J. Mar. Sci. Eng. 2021, 9, 1388. https://doi.org/10.3390/ jmse9121388

Academic Editor: Majid

Mohammadian

Received: 15 November 2021

Accepted: 3 December 2021

Published: 6 December 2021

Publisher's Note: MDPI stays neutral with regard to jurisdictional claims in published maps and institutional affiliations.

Copyright: (c) 2021 by the authors. Licensee MDPI, Basel, Switzerland. This article is an open access article distributed under the terms and conditions of the Creative Commons Attribution (CC BY) license (https:/ / creativecommons.org/licenses/by/ $4.0 /)$.

\begin{abstract}
A turbulent channel flow laden with elongated, fiber-like particles is investigated experimentally by optical techniques. The flow-particle inter-coupling is analyzed in the case of particles with an aspect ratio of 40 and 80 , at two volume fractions, $10^{-5}$ and $10^{-4}$. An image processing technique is presented, which is employed to simultaneously obtain carrier flow velocimetry data and distribution and orientation data of dispersed particles. Turbulence enhancement is reported in the near-wall region, with a higher level of increase associated with higher aspect ratio particles. Comparison to fiber data suggests that this mechanism of turbulence modulation stems from a particles orientational behavior. The preferential particle distribution is reported to be dependent on the aspect ratio in the region close to the wall. The probability density function of the fibers' orientation angle appears to be independent of the particle aspect ratio once it is conditioned to the fibers' characteristic size.
\end{abstract}

Keywords: channel flow; multiphase flow; fibers; non-spherical particles; microplastic

\section{Introduction}

Phenomena of transport and dispersion of non-spherical, anisotropic particles in turbulent flows are ubiquitous in industrial processes and applications. Examples are devices for pollution control, pneumatic transport equipment, pulp and paper manufacturing and wastewater treatment. This class of multiphase turbulent flow is also commonly encountered in many natural processes such as aerosol dispersion in atmosphere, plankton transport in water bodies and sediment re-suspension. Furthermore, micro-plastic pollution in waterways and oceans represents a typical case of dispersion of particles of diverse shapes in a turbulent flow, which can lead to a dramatic impact on the environment.

Turbulent multiphase flows are one of the most challenging fields of fluid mechanics. Based on the degree of interaction between the carrier and the dispersed particle phase, one-way, two-way or four-way coupling can occur. At low volume fractions, the dispersed phase has a negligible effect on carrier flow turbulence, whereas the flow action on the particle phase may lead to the onset of phenomena such as preferential particle concentration and accumulation (one-way coupling). The effect of carrier phase on particle distribution and concentration was investigated by several authors for a range of flow configurations and boundary conditions ([1,2] among others). Particle preferential distribution and orientation has particular relevance for specific applications, such as the papermaking industry, where it is paramount to predict the spatial location and the orientation state of particles within the flow. This aspect dramatically influences the rheological properties of the product and ultimately its quality. The scenario changes at higher particle volume fractions. Under these conditions, momentum exchange between phases (two-way coupling) gradually increases. Effects of turbulence modulation of the carrier phase (enhancement or reduction) may be associated with this flow regime. Notwithstanding the impact on the industrial applications, the understanding of turbulence modulation by dispersed particles 
represents a hot topic in the research of turbulence fundamentals. In the last decades, several works analyzed turbulence modulation by dispersed particles ([3-10]). As the volume fraction of dispersed particles increases, inter-particle effects start to occur and four-way coupling between phases occurs. This regime will not be discussed in this manuscript.

Most of the literature on turbulent multiphase flows deals with spherical particles. In the case of non-spherical particles dispersion, flow-particle interplay is even more challenging. Single particle orientation in three-dimensional space plays a pivotal role as it affects torques and forces exerted by the flow on the particle itself. Elongated particles, alternatively named rod-like or fiber particles, are an effective model to investigate the behavior of anisotropic particles in turbulent flows. Furthermore, they are a practical example, which is found in many applications and processes, such as papermaking applications, ice crystal formation in clouds and micro-plastic pollution.

The dynamics of rod-like fibers in shear flows have been theoretically predicted by [11]. According to the theory, and with the limitation of shear flow conditions, rod-like particles tend to align mostly with the mean flow, although they undergo a periodic revolving motion according to the so-called Jeffery orbits. Recently, the interest in the study of anisotropic particles behavior in turbulent flows has grown considerably (refer to [12] for a comprehensive review on the subject). Several authors focused on fiber particles behavior in a range of turbulent flow configurations by experiments and simulations [13-16]. Recent works have investigated specific aspects such as the rotational dynamics of elongated particles and the behavior of non-axisymmetric particles in channel flow [17-19]. The role of fiber length on the fiber dynamics in a turbulent channel flow was investigated experimentally by [20]. Drag-reduction by non-spherical particles was investigated numerically by [21-23] in turbulent channel flows. The majority of research on elongated particle dynamics in turbulent flows is focused on the analysis of the dispersed phase behavior, namely on the effect of the carrier flow on the dispersed phase.

In order to characterize and possibly predict the turbulence intensity modulation due to the particle dispersion, Gore et.al [24] proposed a simple approach based on particle diameter and turbulent flow of large eddies. The underlying rationale is that the drag force on particles whose diameter is smaller than the most energetic eddies dampens the turbulence intensity, whereas particles larger than the most energetic turbulent eddies produce wakes that can increase turbulence. Nevertheless, this approach is based on the assumption of spherical dispersed particles. Elongated particles entail more complex behavior, as their impact on the flow features is supposedly dependent not only on particle volume fraction but also on the orientational status of the fibers within the flow. As a matter of fact, there is still a lack of data on the flow-particle coupling and the impact on turbulent characteristics of the carrier phase of elongated particle dispersion and transport.

Experimental analysis of elongated, fiber particles suspensions poses significant difficulties. In particular, the use of optical techniques is limited by the maximum observable mass fractions. Nonetheless, the valuable information that can be potentially obtained by optical methods, in terms of simultaneous identification of fiber orientation and velocity measurement of the surrounding fluid, represents a distinct advantage over other techniques and has represented the main drive towards the choice of this approach. Among optical techniques, Particle Image Velocimetry, hereafter PIV, has proved to be the most effective choice compared to other methodologies, i.e., Laser Doppler Anemometry (LDA), since it allows simultaneous measurement of dispersed and carrier phase velocity. Usually, for dilute flows, measurement of the particle-phase velocity field does not pose difficulties if dispersed particles are larger enough than the tracer particles, as they generate brighter images and can be effectively identified [25]. Measurement of carrier phase velocity is more difficult, and ad hoc discrimination techniques to separate carrier phases from dispersed ones are necessary. While in applications where the carrier phase is gaseous the difference in sizes between tracers and dispersed particles usually allows phase separation based on size or intensity/size maps [25], liquid/solid multiphase flows require more sophisticated approaches. Such methodologies may be roughly split into two categories. Two-camera 
schemes typically rely upon the use of a fluorescent tracer, which emits light at different wavelengths than the light source. With appropriate optical filters, two cameras can image the dispersed phase Mie scattering and the fluorescent emission of the carrier phase separately. An example of such approach is found in [26]. Single camera approaches are intrinsically easier to implement and less expensive. A review of single-camera phasediscrimination techniques is found in [25]. Past works focused on the estimate of the modulation of turbulent kinetic energy dissipation rate by particles [27] and measurement of the carrier phase velocity field close to particles surface [28,29]. In [9], time-resolved PIV was employed to analyze the interactions of spherical dispersed particles with the near-wall coherent structures in a turbulent channel flow. More recently in [30], PIV and Particle Tracking Velocimetry (PTV) were respectively used to analyze a particle-laden turbulent channel flow in a wide range of Reynolds numbers for a range of spherical particle diameters. As regards elongated particles, dilute fiber suspensions at low Reynolds number were investigated in [31-33] in different configurations. The authors reported a strong influence of fluid turbulence intensity and wall-distance on fiber orientation and accumulation. Nonetheless, in all of these works the carrier fluid velocity field is not measured and thus analysis on the coupling between the flow and dispersed phase is not provided.

The aim of this work is to analyze the features of a turbulent water channel flow laden with two types of anisotropic rod-like, fiber particles with different aspect ratios. The rod-like particle suspensions are investigated at two different volume fractions. Carrier flow velocimetry data are analyzed in order to highlight turbulence modulation effects exerted by the particles onto the carrier flow with respect to the clear-water reference case. The preferential accumulation and orientation of dispersed particles is also investigated so that a comprehensive picture of the flow-particle coupling taking place is provided. The role played by the fiber aspect ratio in the coupling between the carrier flow and dispersed phase is then analyzed and discussed.

\section{Materials and Methods}

\subsection{Experimental Set-Up}

Experiments were carried out in a turbulent channel flow facility, which consists of a rectangular section channel $140 \mathrm{H}$ long, $2 \mathrm{H}$ high and $20 \mathrm{H}$ wide, where $\mathrm{H}=1 \mathrm{~cm}$ is the half channel height. In order to reduce turbulence intensity within the test section, the rectangular section is preceded by a 10:1 contraction and by a $5 \mathrm{H}$ long flow straightener module. The latter also serves the purpose of further increasing flow uniformity. The contraction section is shaped based on a polynomial profile in order to prevent flow separation and is $20 \mathrm{H}$ long. According to the literature findings [2], the channel width ensures that the flow can be reasonably considered on average as two-dimensional in the XY plane, parallel to the main flow direction. The test section is made of plexiglass so that full optical access from all directions is possible. The flow is gravity-driven by a reservoir tank $55 \mathrm{H}$ high, which is supplied by a submerged pump sitting inside a stagnation chamber, where the channel discharges. Schematics of the experimental set-up are provided in Figure 1. Images were collected with a standard cross-correlation CCD camera (PCO Pixelfly QE, 14-bit dynamic range, $1392 \times 1024$ pixels image resolution). The camera lens used for all acquisitions is a Nikon F $50 \mathrm{~mm}$ in focal length, with a maximum aperture of 1.2, equipped with a $10 \mathrm{~mm}$ extension tube. Laser lighting was provided by a Nd:YAG double cavity unit $(200 \mathrm{~mJ}$ per pulse at $12.5 \mathrm{~Hz})$ for an estimated laser sheet thickness of $2 \mathrm{~mm}$. A mirror is used to redirect the laser sheet to the acquisition plane. Fluid was seeded with neutrally buoyant silver-coated $10 \mu \mathrm{m}$ diameter hollow glass spheres (Dantec HGS-10). For the dispersed phase, nylon fibers (Polyamide 6.6, density $1.13 \mathrm{~g} / \mathrm{cm}^{3}$ ) were employed, with two different mean lengths: $\mathrm{L}_{1}=2 \mathrm{~mm}$ and $\mathrm{L}_{2}=4 \mathrm{~mm}$. Mean diameter is $\mathrm{d}=50 \mu \mathrm{m}$ for both sizes so that the fiber aspect ratios are, respectively, $k_{1}=\mathrm{L}_{1} / \mathrm{d}=40$ and $k_{2}=\mathrm{L}_{2} / \mathrm{d}=80$. 

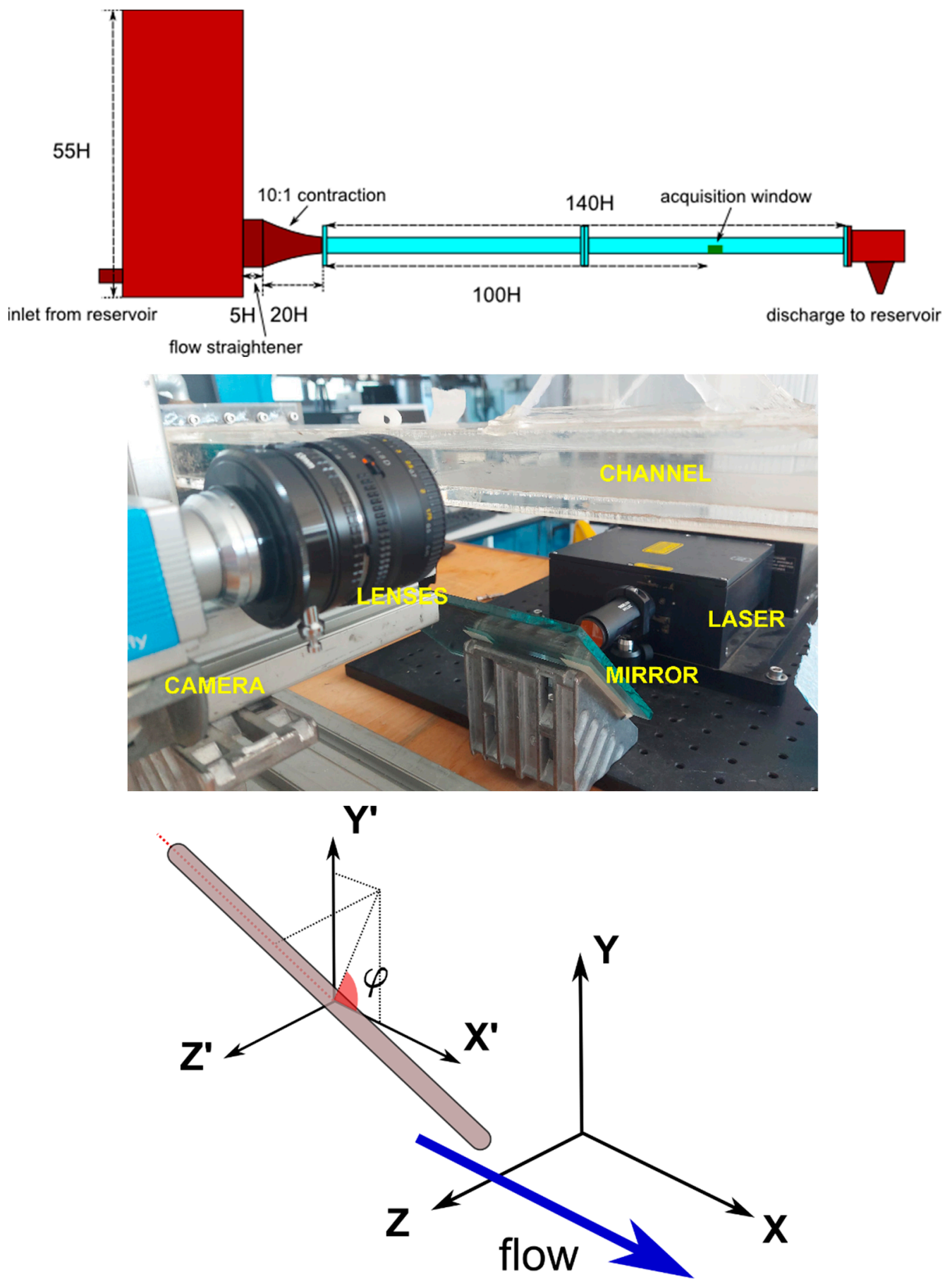

Figure 1. Schematics (top) and picture (middle) of the channel flow experimental set-up. Elements are not to scale. Representation of flow frame of reference $(X, Y, Z)$, particle centered co-moving, non-rotating frame of reference $\left(X^{\prime}, Y^{\prime}, Z^{\prime}\right)$ and in-plane particle orientation angle (bottom).

The acquisition plane is perpendicular to the channel bottom wall, parallel to the channel side walls and is located at the channel centerline. Images were acquired at $100 \mathrm{H}$ downstream of the channel inlet, in a region where the flow can be deemed as fully developed. Schematics of the flow measurements' reference of frame $(X, Y, Z)$ is provided in Figure 1 along with a generic fiber, for which a local, non-rotating co-moving coordinate system $\left(X^{\prime}, Y^{\prime}, Z^{\prime}\right)$ is shown, centered in the fiber's centroid. The acquisition campaign consists of five test cases; a reference clear-water case and four cases for the fiber-laden flow. For each kind of the two fiber aspect ratios, $k_{1}$ and $k_{2}$, two different volume fractions 
were investigated, namely $C_{\text {low }}=10^{-5}$ and $C_{\text {high }}=10^{-4}$. The flow suspension was obtained by injecting a given weighted mass of fibers in the known, constant fluid volume. We point out that an uncertainty of approximately $1 \%$ should be allowed for in the mentioned volume fraction concentrations due to the accumulation of fibers throughout the apparatus during operations.

The size of the imaged area for all acquisitions is $1.1 \mathrm{H} \times 1.5 \mathrm{H}$, with a spatial resolution of $0.01 \mathrm{~mm} /$ pixel. For each test case, 10,000 image pairs were acquired so that higher-order statistics of the fluid velocity field are available. The acquisition frequency was set to $13 \mathrm{~Hz}$, and the time between frames was set to $80 \mu \mathrm{s}$.

\subsection{Carrier Flow Velocity Field Measurement}

Planar flow velocity fields in the clear-water case and fiber-laden cases were obtained via planar PIV. The velocity field $(u, v)$ was calculated with the commercial software Insight $4 \mathrm{G}$ by TSI, Inc. Images were background-subtracted and fed to the cross-correlation algorithm. The algorithm implements an iterative multi-pass, multigrid, image deformation scheme with window-offset. The PIV interrogation window size was set to $64 \times 64$ pixels for the first pass and $32 \times 32$ pixels and $16 \times 16$ pixels for the second and final passes. The resulting vector grid spacing is 8 pixels based on a subwindow overlap of $50 \%$, which corresponds to approximately $0.01 \mathrm{H}$, corresponding to $0.1 \mathrm{~mm}$. In the validation step, spurious vectors are dropped according to a local median test carried out on a 9-point square kernel. Table 1 summarizes the main parameters of the current experimental setup compared to reference data from $[19,34]$. Respectively $U_{0}, u_{\tau}, \operatorname{Re}_{H}, \operatorname{Re}_{\tau}$ represent the mean centerline channel velocity, the friction velocity, the Reynolds number based on the centerline velocity $R e_{H}=U_{0} \mathrm{H} / v$, and the friction Reynolds number $R e_{\tau}=u_{\tau} \mathrm{H} / v$, where $v$ is the kinematic viscosity of water at $20{ }^{\circ} \mathrm{C}$.

Table 1. Channel flow main characteristic data.

\begin{tabular}{ccccc}
\hline Work & $\boldsymbol{U}_{0}(\mathrm{~m} / \mathbf{s})$ & $\boldsymbol{u}_{\boldsymbol{\tau}}(\mathrm{m} / \mathbf{s})$ & $\boldsymbol{R e}_{\boldsymbol{H}}$ & $\boldsymbol{R} \boldsymbol{e}_{\boldsymbol{\tau}}$ \\
\hline current & 1.1 & 0.0585 & 11,000 & 530 \\
Alipour et al. (2021) [19] & 0.172 & 0.00102 & 6250 & 360 \\
Kasagi et al. (1995) [34] & 0.13 & 0.00684 & 5540 & 290 \\
\hline
\end{tabular}

\subsection{Image Processing}

Multiphase flow images require sophisticated methods in order to discriminate between images from flow tracers and dispersed phases. Early methods rely upon high-pass filters and intensity-size maps to carry out phase separation [25,35], but more advanced methods are required when particles with complex shapes are under scrutiny, such as in the current experiment. The typical approach to the phase discrimination problem entails the generation of two images from every single multiphase image; one having only the flow tracers and the other showing only the fiber particles. These two datasets are then further processed to obtain separate information on carrier flow velocity field and fibers location, distribution and orientation within the flow. In the current work, we follow a different approach, which consists of the generation of a masking fiber image from each multiphase image. This masking image, which contains only fibers particles, is used in the PIV process to mask the dispersed particles so that only the remaining flow tracers are considered for the calculation of the carrier flow velocity field. The masking image is then used to identify fiber particles' location, distribution and orientation within the flow. This approach, by using the same particles' image to extract particle information and to mask the original two-phase image, has the advantage of not requiring the generation of a tracers-only image, thus expediting the separation process. Furthermore, the downside of the generation of a tracers-only image is to create black areas where dispersed particles were located, possibly affecting the PIV cross-correlation step. The processing steps may be summarized as follows: 
1. Background-subtraction is carried out in order to remove noise from steady sources such as wall reflections, background illumination.

2. A spatial median filter is applied to the images (3 pixels square filter size). The effect of the latter step on the pre-processing of multiphase images is thoroughly discussed in [35].

3. The images obtained in the previous step are binarized. The presence of fibers affects the local grey level intensity, because the area surrounding the fibers is brighter due to the increased light scattering. This effect is accounted for by applying a moving intensity threshold to each image. Each picture is divided into smaller tiles for which a local threshold based on the grey level average and standard deviation is calculated. Each pixel exceeding the local average more than one standard deviation is set to zero.

4. Morphological operations of erosion and dilation are carried out. The operations of erosion and dilation, respectively, remove and add pixels to the boundary of each interconnected region of pixels. The erosion removes outstanding tracer images, whereas the subsequent dilation enlarges the fiber images, so that they can be used as masks in the next steps.

5. Each masking image is processed to obtain information on dispersed fibers, as described in the next section. The fiber image obtained is employed as a masking image for the next cross-correlation step.

6. PIV velocity fields $(\mathrm{u}, \mathrm{v})$ are obtained for each instantaneous image by applying the fiber masking image obtained in the previous step. Cross-correlation is carried out on tracer images only.

Processing and separation steps are summarized in Figure 2 where a region of a raw image is provided along with an image after pre-processing steps 1 and 2, binarization step 3 and morphological transformation step 4 . A sample of an instantaneous carrier flow velocity field is shown in Figure 3 overlaid onto the original acquired image. The masked region is highlighted in red shade.
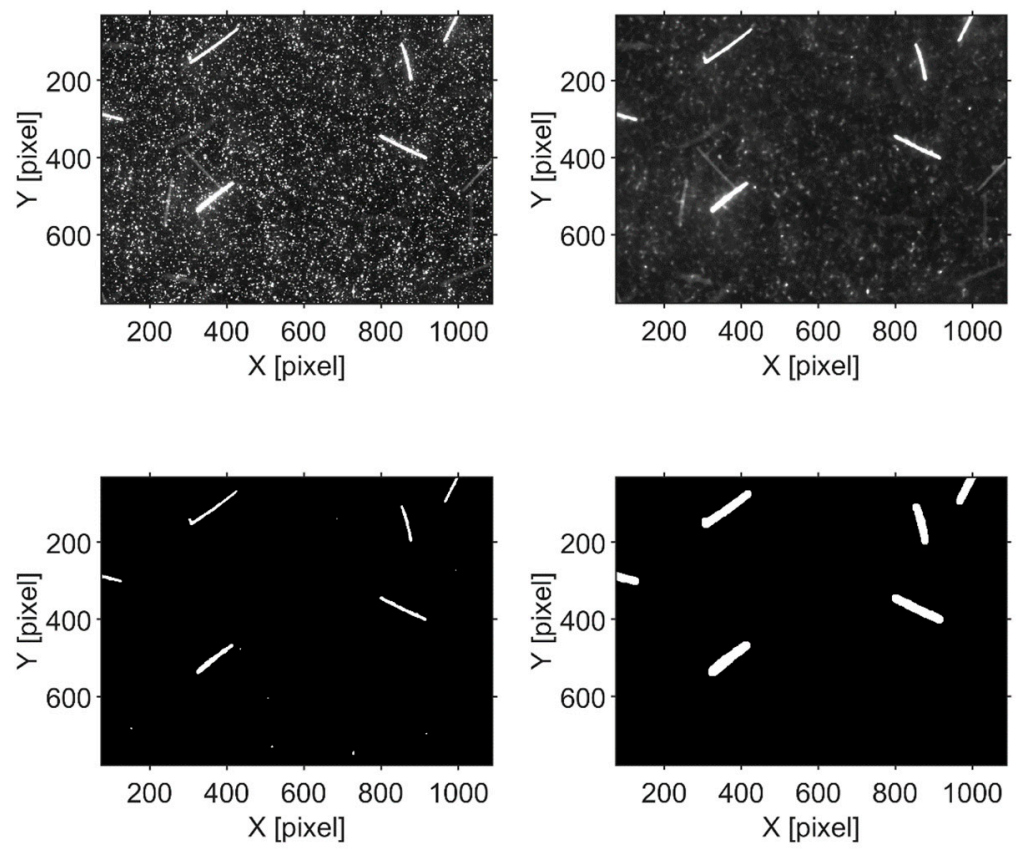

Figure 2. Processing and separation steps. Original raw image (top left). Image obtained after background subtraction and median filter application (top right). Binarized image (bottom left). Final mask image obtained by erosion and dilation operations (bottom right). 


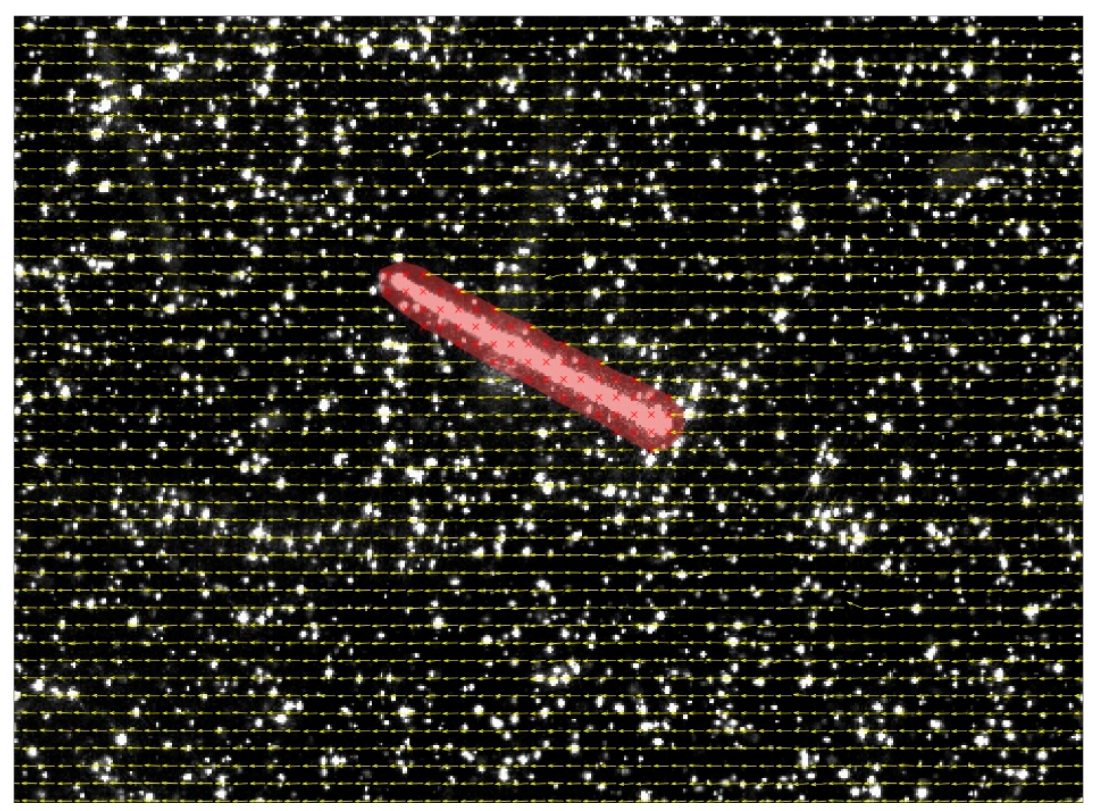

Figure 3. Instantaneous vector field surrounding a fiber (enlarged region) overlaid onto the original acquired image. Highlighted in red is the calculated fiber mask. $C_{\text {low }}$ and $k_{1}$ case.

\subsection{Fibers Data Extraction}

Masking images obtained via the image processing step are used to extract information on dispersed fibers. Connected image regions are labeled as fibers according to their eccentricity and area size. If the aspect ratio exceeds a set threshold (set here as 2) and the area is higher than three-times the tracers' diameter, the particle image is considered as a fiber. For each particle image identified as a fiber, a set of information is retrieved-area, particle centroid location in the acquisition plane, and orientation of the major axis of the fitting ellipse. The latter is obtained based on a third-order least square approach. From this set of data, statistics of fiber preferential distribution and orientation within the turbulent flow are obtained.

This approach is limited by the fact that the proposed technique is based on a twodimensional approach, since the acquired images are the two-dimensional projection on the camera sensor of the volume of fluid illuminated by the laser sheet. This is a common approach for the study of unladen channel flow provided that based on the channel span-wise depth, two-dimensionality is considered acceptable. On the other hand, the assessment of this method on the analysis of the behavior of three-dimensional objects such as rod-like fibers is necessary. The approximation introduced in this work is based on the observation that only the in-plane orientation of fiber particles is detected, whereas the full spatial orientation state cannot be determined. This implies that fibers parallel to the span-wise axis $Z$ could be erroneously identified as fluid tracers since their projection would be almost circular. These fiber images are thus ruled out by the separation algorithm leading to a certain amount of missed fiber detection. With this regard, based on the findings from our previous works [14], approximately $0.5 \%$ of all fibers are expected to be missed.

Consolidated fibers data are then manipulated in order to extract statistical information on fiber accumulation and orientation state. The vertical axis $Y$ is divided into a number of slots of 32 pixels width and for each slot, fibers whose centroid location lies within the slot limits are considered. Thus, for each set, the mean and root mean square (RMS) of the in-plane orientation angle $\varphi(Y)$ are calculated. Furthermore, the relative concentration of fibers is measured. The slot size along the vertical axis $Y$ is determined based on a trade-off between the size of the data sample for each slot and the resulting resolution. 


\section{Results}

\subsection{Clear Water Case}

In this section, results from the clear-water reference case are provided in order to assess the flow quality and to validate the experimental set-up. As described in the previous section, the whole data set consists of $\mathrm{N}=10,000$ image pairs. Flow stability over acquisition time was evaluated by comparing results obtained with sets of 2000 image pairs. Mean velocity profiles confirmed a variability between each set of less than $1 \%$. In Figure 4 , the average stream-wise velocity $U$ along the vertical coordinate normalized with respect to the friction velocity $u_{\tau}=0.0585 \mathrm{~m} / \mathrm{s}$ is shown. The friction velocity is determined via the Clauser method using the universal Spalding profile with $\mathrm{k}=0.41$ and $\mathrm{B}=5.2$ and plotted versus normalized wall units $y^{+}=u_{\tau} \mathrm{y} / v$. The uncertainty of the mean profile is below $0.5 \%$ with a confidence interval of $95 \%$ based on Student's t-distribution. Data are compared to experimental data from the literature and to the logarithmic law of the wall. The velocity profile trend confirms that the flow is fully developed in the acquisition region. Flow stability along the stream-wise $\mathrm{X}$ coordinate is also assessed by comparing the velocity profile at different $X$ locations. Results, not shown here, indicate a variability of less than $0.5 \%$.

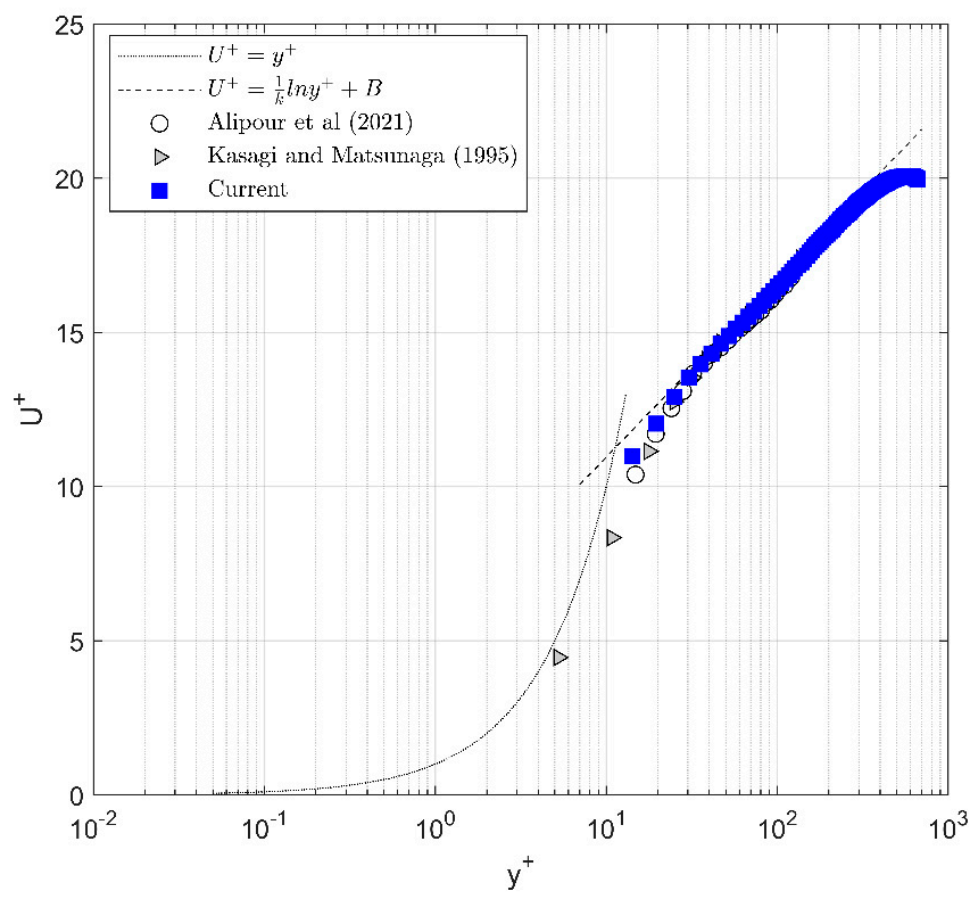

Figure 4. Clear-water reference case. Average stream-wise profile in wall coordinates compared to logarithmic law of the wall $(K=0.41$ and $B=5.2)$ and reference data from Alipour et al. (2021) and Kasagi and Matsunaga (1995).

\subsection{Fiber-Laden Cases}

\subsubsection{Carrier Flow}

In this section, the carrier flow velocity statistics are analyzed in order to shed light on the turbulence modifications that arise in the presence of elongated dispersed particles. In Figures 5 and 6, the stream-wise average velocity profile and root mean square of streamwise and vertical components of the carrier flow velocity field are, respectively, shown for the clear-water case and fiber-laden cases with aspect ratios $k_{1}$ and $k_{2}$. The uncertainty of the mean profile is below $0.5 \%$ with a confidence interval of $95 \%$ based on a Student's $\mathrm{t}$-distribution. The particles volume fraction corresponds to the lowest concentration $C_{\text {low }}$. Carrier flow turbulence enhancement is reported, in particular in the near-wall region of the channel. This confirms that at this particle volume fraction two-way coupling is 
present, i.e., the momentum transfer from the dispersed phase towards the carrier phase is not negligible [36]. A relevant role appears to be played by the fiber aspect ratio, with a larger increase in turbulence fluctuations associated with higher values. Observation of trends reveals that the region of highest enhancement of turbulence is that below 40 wall units approximately. In this region, the profiles of aspect ratio $k_{1}$ and $k_{2}$ diverge the most, suggesting that in the near-wall region, the mechanism underlying the increase in turbulent fluctuations of the carrier flow is strongly linked to the particle's aspect ratio. One of the mechanisms of turbulence enhancement by dispersed particles is described by [3] as related to enhanced velocity fluctuations due to wake dynamics and self-induced vortex shedding by the dispersed particles. Other authors [37] have suggested that close to the wall, elongated particles undergo a dynamic phenomenon called pole vaulting, during which they rapidly rotate and change their orientation attitude. The occurrence of this phenomenon, associated with wake vortex generation by the rotating fibers, could explain the reported dependence on the fiber's aspect ratio. Higher rotation rates of fibers in the near-wall region were also reported in other literature works confirming this scenario. Observation of average velocity profiles shows that in the flow region matching the turbulence enhancement, the carrier flow in the fiber-laden cases is characterized by reduced speed, as reported in Figure 5. However, as opposed to the turbulent fluctuations, the fiber aspect ratio does not appear to play a role, as the trends are substantially equal. The observation that the effect of aspect ratio is limited to the fluid fluctuations points again to the role played by the different dynamic behavior exhibited by the fiber in the near-wall region. The impact of the dispersed fibers onto the carrier flow turbulence is further investigated in Figure 7, where the RMS of stream-wise and vertical fluctuations, $\mathrm{u}^{\prime}$ and $\mathrm{v}^{\prime}$, normalized to the centerline velocity $U_{0}$ are depicted for the $C_{\text {low }}$ case. Similar to the horizontal component, the vertical component appears to be affected by the dispersed fibers, with the vertical component fluctuations enhanced in the region approximately up to $\mathrm{Y} / \mathrm{H}=0.5$. It descends that the action exerted by the fibers onto the flow is primarily associated with the rotational dynamic of the fibers, rather than translational motion. In fact, this latter motion is mostly in the horizontal, stream-wise direction, as shown by several authors $([14,19]$ among others).

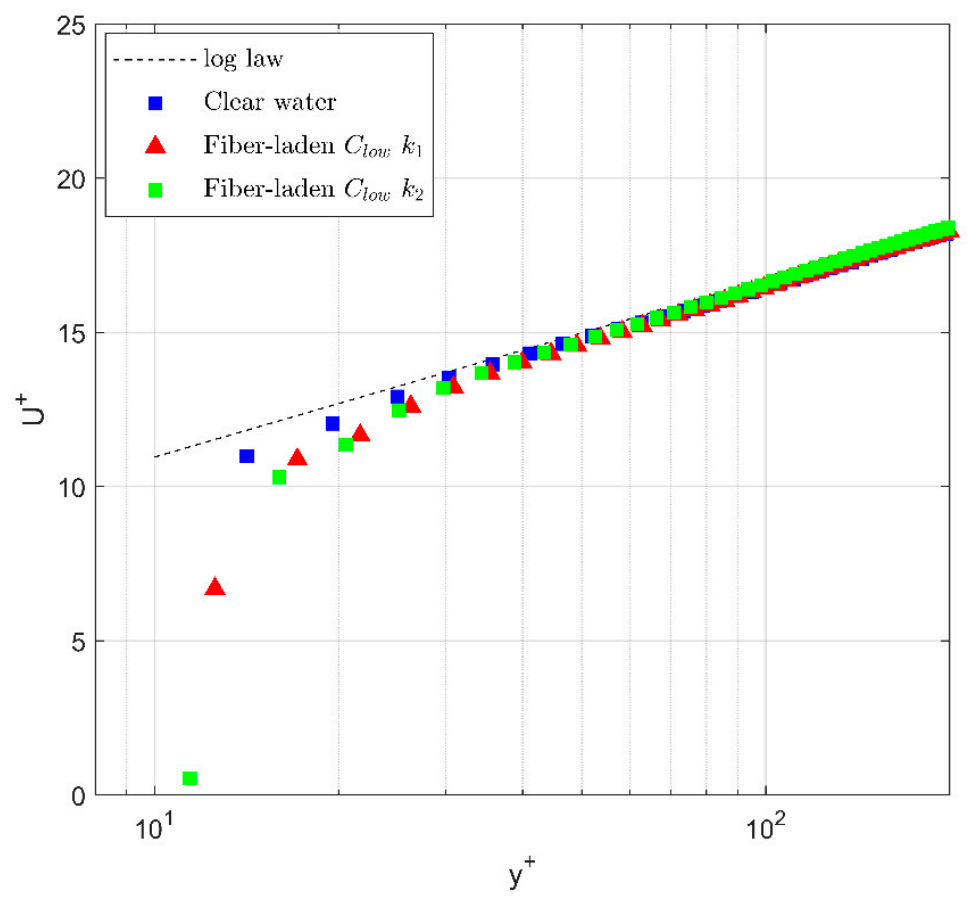

Figure 5. Carrier flow average stream-wise profile in wall coordinates. Clear-water case compared to the fiber-laden case at low concentration $C_{\text {low }}$, two aspect ratios $k_{1}$ and $k_{2}$. 


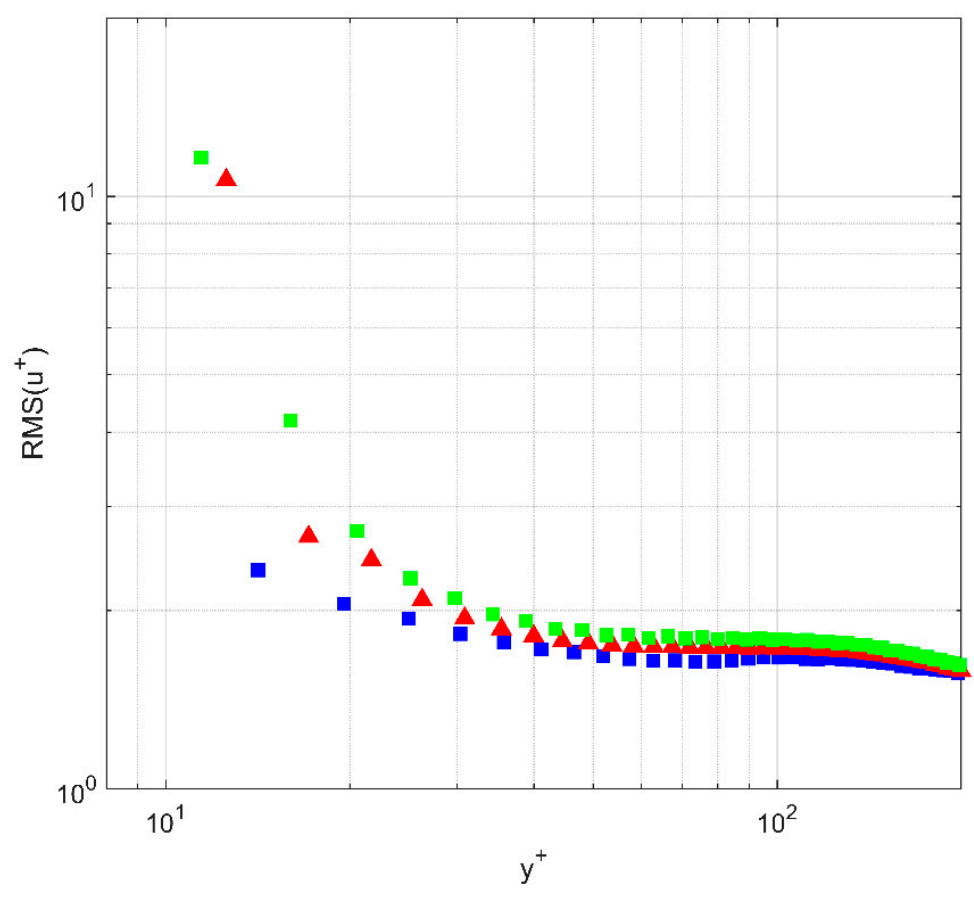

Figure 6. Carrier flow root mean square of stream-wise component of velocity in wall coordinates. Clear-water case compared to the fiber-laden case at low concentration $C_{\text {loww }}$, two aspect ratios $k_{1}$ (red triangles) and $k_{2}$ (green squares).
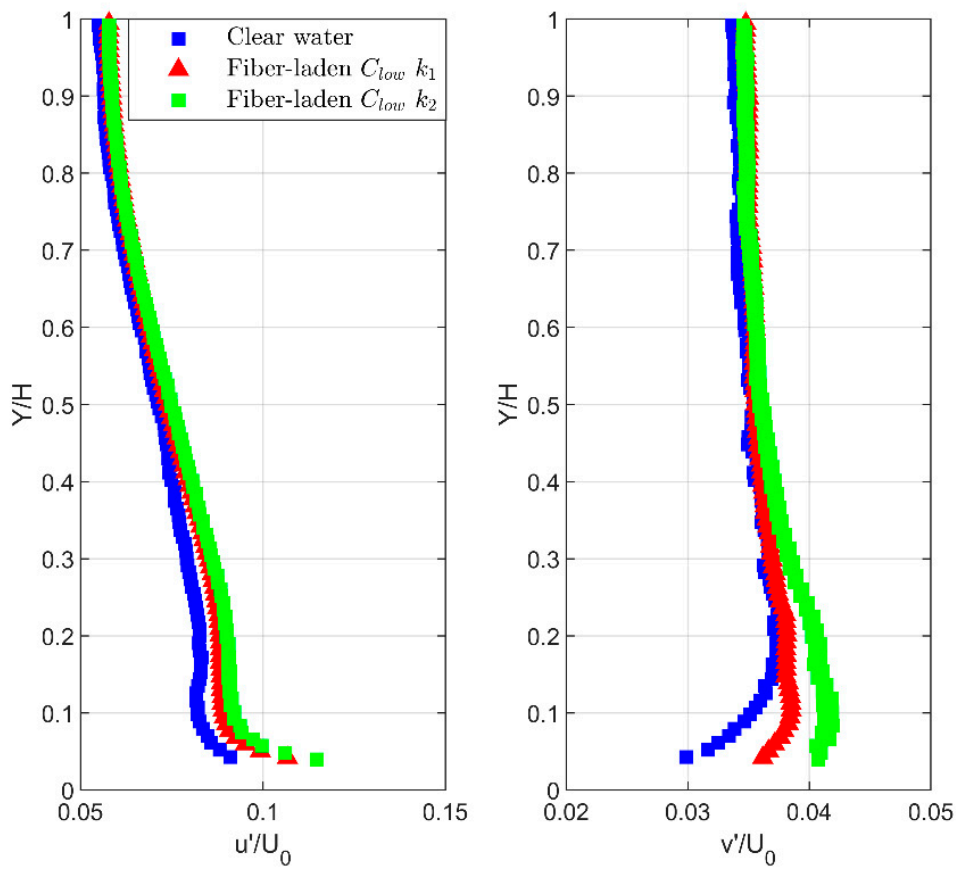

Figure 7. Carrier flow root mean square of stream-wise (left) and vertical (right) components of velocity. Clear-water case compared to the fiber-laden case at low concentration $C_{\text {low, }}$, two aspect ratios $k_{1}$ (red triangles) and $k_{2}$ (green squares).

In Figure 8, the same quantities are shown in the case of high particle concentration. As expected, the degree of turbulence modulation increases in the case of a larger volume fraction and extends further away from the wall than the low concentration case. The vertical component fluctuations appear to undergo a higher relative increase than the horizontal one. The role of fiber aspect ratios appears unchanged with respect to lower 
volume fraction suspension, with an increase in turbulent fluctuations, which is most evident in the near-wall region.
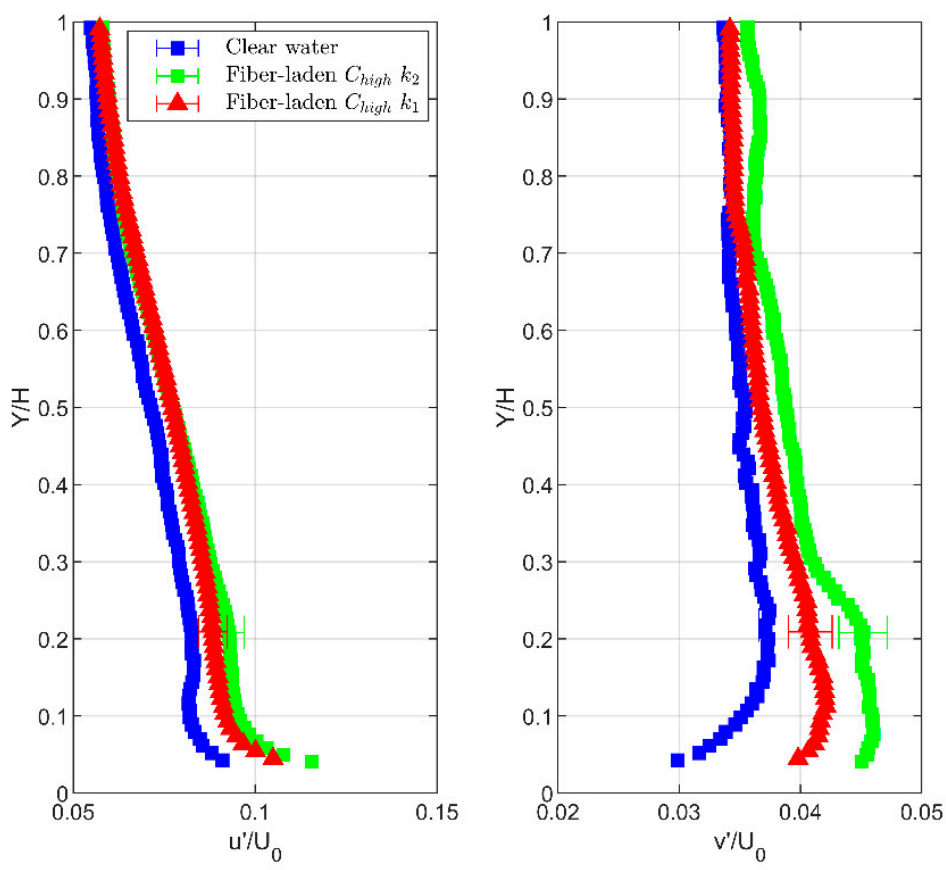

Figure 8. Carrier flow root mean square of stream-wise (left) and vertical (right) components of velocity. Clear-water case compared to the fiber-laden case at high concentration $C_{\text {high }}$, two aspect ratios $k_{1}$ (red triangles) and $k_{2}$ (green squares). The 95\% confidence error bars shown.

Additional information about the turbulence modulation effects is obtained from the inspection of higher-order statistical moments of velocity fluctuations. The third statistical moment $\mathrm{u}^{\prime \prime}$ and $\mathrm{v}^{\prime \prime}$ (skewness) of carrier flow fluctuations is shown in Figure 9 for the unladen reference case and fiber-laden cases, for the two aspect ratios, at low concentration. Values are normalized versus $U_{0}{ }^{3}$. A negative value of $\mathrm{u}^{\prime \prime}\left(\mathrm{v}^{\prime \prime}\right)$ means that horizontal (vertical) fluctuations are more likely to take on large negative values than large positive values.

While the vertical component profiles do not feature relevant differences for the two aspect ratios, the stream-wise component shows lower values of skewness for the high aspect ratio case in the region $0.1 \mathrm{H}<\mathrm{Y}<0.8 \mathrm{H}$. This occurrence points out that in the region outside the near-wall area there is a higher probability of measuring high peak values of stream-wise fluctuations as the fiber aspect ratio increases. A similar trend is observed for the highest concentration case but is not reported here.

\subsubsection{Dispersed Particles}

In this section, the statistics of fiber accumulation and orientation are analyzed. Fiber particles data are processed according to the slotting approach set out in Section 2.4. In Figure 10, the relative concentration of fibers $\mathrm{N}$ within the channel in the wall coordinates is shown for all the fiber-laden cases analyzed. This value is normalized with respect to center channel concentration. For both aspect ratios, the concentration trend features a region of increasing accumulation starting from the wall and progressing up to a stable value matching the channel centerline value. Fibers with lower aspect ratio $k_{1}$ exhibit a region of increasing concentration, which extends up to approximately $y^{+}=10$. The relative concentration then increases to its maximum value, at approximately $y^{+}=25$, before dropping again, leveling at a steady concentration value from approximately $y^{+}=70$ and on. This trend is compared to the simulations presented by [38], obtained for elongated particles with aspect ratios similar to the current data. The preferential accumulation of fibers in the near-wall region within one fiber length from the wall is explained by [39] 
with the difficulty undertaken by fibers to escape this high-speed flow region resulting in a tendency to spend a relatively longer time in this flow area. Results in Figure 10 confirm this phenomenon for $k_{1}$ fibers, for which fiber length $\mathrm{L}_{1} \approx 30 y^{+}$. On the other hand, fibers with a higher aspect ratio exhibit an almost linear increase up to nearly $y^{+}=70$, where they reach the centerline value, apparently not being affected by the bounding effect due to the near-wall high-speed region. The ability exhibited by particles to escape the near-wall area appears to be dependent on the aspect ratio, in that higher values are supposedly more easily ejected towards the outer flow region by pole-vaulting induced by the flipping motion close to the wall. We point out that aspect ratio appears to affect the homogeneity of particle distribution in the far-wall and centerline regions. In fact, the higher aspect ratio of fiber concentration features a "jagged" profile compared to the smoother one of the lower aspect ratio particles. Interestingly, such an irregular profile was reported by [32], where this phenomenon was associated with flocculation by fibers. Although the volume fractions tested are not high enough to trigger such events, it is suggested that longer fibers could be more prone to local aggregation events. Furthermore, the volume fraction of the multiphase suspension does not appear to affect the way dispersed particles accumulate within the channel. Cosine of average in-plane angle of fibers $|\cos \varphi|$ is presented in Figure 11, compared to experimental data from [19], with elongated particles with an aspect ratio of 30.7. Close to the wall, fiber particles tend to align on average to the main flow direction, whereas as they depart from the near-wall region, they tend take on the centerline orientation. The preferential alignment region extends up to the logarithmic region of the turbulent boundary layer, with very similar behavior for the two volume fractions. While the general trend is retained, the fiber's aspect ratio plays a role in the actual orientation angle values of elongated particles, which is reported to be slightly lower for $k_{1}$ fibers. A comparison to carrier flow turbulence fluctuations data provided in Figures $6-8$ reveals that the near-wall region up to $100 y^{+}$, where the carrier flow turbulence is increased by particle presence, matches the channel area where fibers tend to be more aligned with the stream-wise direction $X$. This occurrence can be explained by inspection of RMS of the fibers' orientation angle, provided in Figure 12. It is reported that close to the wall, fluctuations of the fibers' orientation angle exceed the centerline value by up to $10 \%$, for the high aspect ratio particles $k_{2}$, with the same trend observed for all the test cases. With this respect, lower aspect ratio fibers are associated with the lower fluctuations of the orientation angle, whereas the particles' volume fraction has a reduced impact on the curves. These findings suggest that the main mechanism through which momentum transfer takes place is dependent directly on fiber orientation status. Relative concentration of fibers within the flow appears to act in an indirect way on turbulence modulation in that lower relative concentrations would make it easier for the particles to rotate freely compared to flow regions where fibers accumulate more.

The capability of elongated particles to freely rotate and take on a particular orientation is dependent on their location within the flow and in particular with respect to the channel wall. As shown in Figure 10, fibers in the proximity of the wall are most likely to align to the stream-wise direction of the flow due to the constraint represented by the wall proximity.

As the wall constraint is relaxed, i.e., fibers are far from the wall, they are free to rotate and orientate under the effect of the carrier flow. In order to shed light on this phenomenon, we estimate the probability of the fiber orientation angle $\operatorname{PDF}(\varphi)$ conditioned to the fiber location within the channel. In Figure 13, we show the estimate of the probability density function conditioned to the location of the fibers, namely below $\left(\mathrm{y}<\mathrm{L}_{1,2} / 2\right)$ or over $\left(y>L_{1,2} / 2\right)$ half fiber length. Data are provided for both tested aspect ratios and for a high-volume fraction case. It is evident that as particles centroids are located below half fiber length, particles will most likely orientate parallel to the $X$ axis and to the wall. Particles farther from the wall than the fiber half-length are no longer subject to the wall constraint and therefore feature a flatter PDF since they are free to orientate. We point out that the PDFs shown are not symmetric, which is in agreement with the mean orientation results discussed previously. In fact, the most likely value, which is 
different from $\varphi=0^{\circ}$, matches the results depicted in Figure 11, and lies in the range $16^{\circ}<|\varphi|<25^{\circ}$. Comparison between the two aspect ratios conveys that conditioned PDFs are very similar. This notable result suggests that the statistical distribution of the orientation state of elongated particles in the presence of the wall collapses when they are scaled according to their characteristic size.
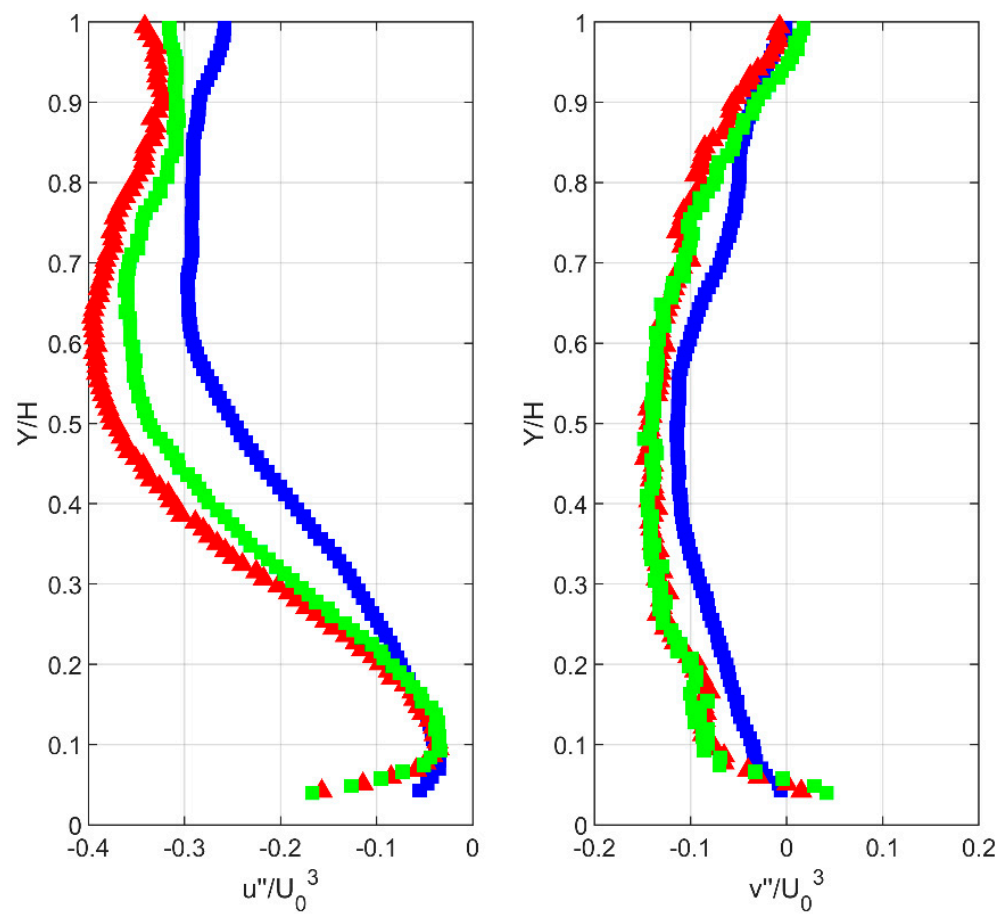

Figure 9. Carrier flow third moment of stream-wise (left) and vertical (right) components of fluctuating velocity. Clear-water case compared to the fiber-laden case at low concentration $C_{\text {low }}$, two aspect ratios $k_{1}$ (red triangles) and $k_{2}$ (green squares).

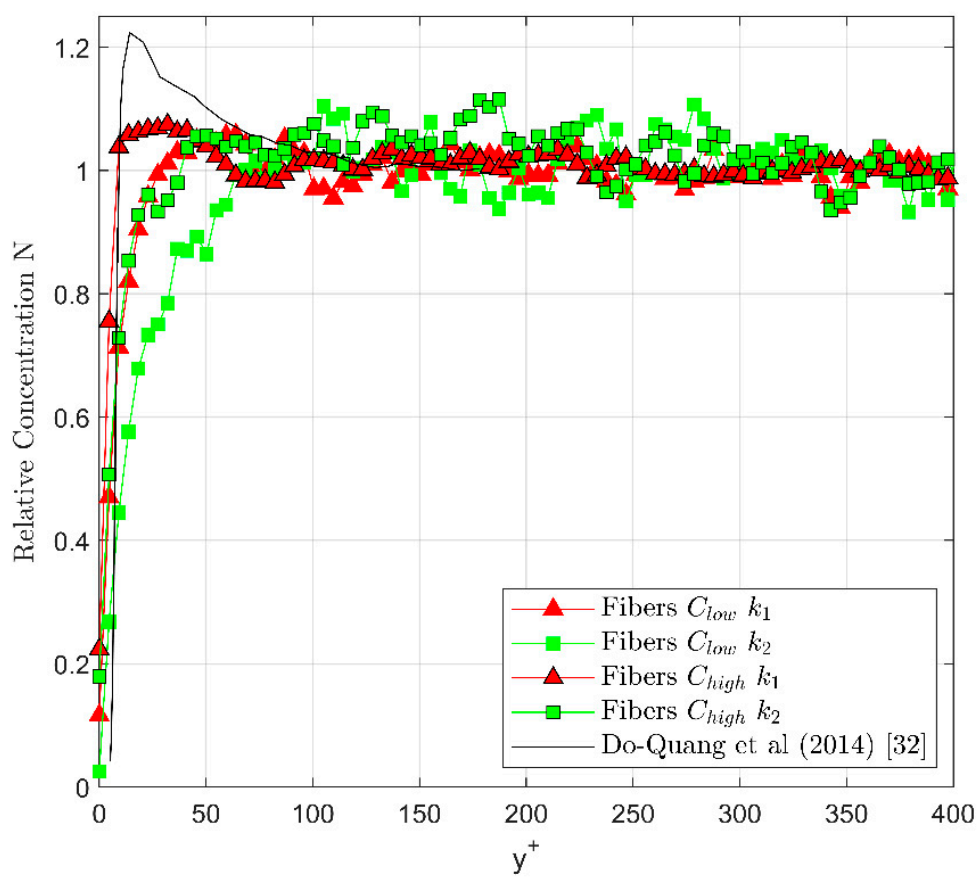

Figure 10. Relative concentration $\mathrm{N}$ of fiber particles normalized versus centerline concentration. Data compared to Do-Quang et al. (2014) [38]. 


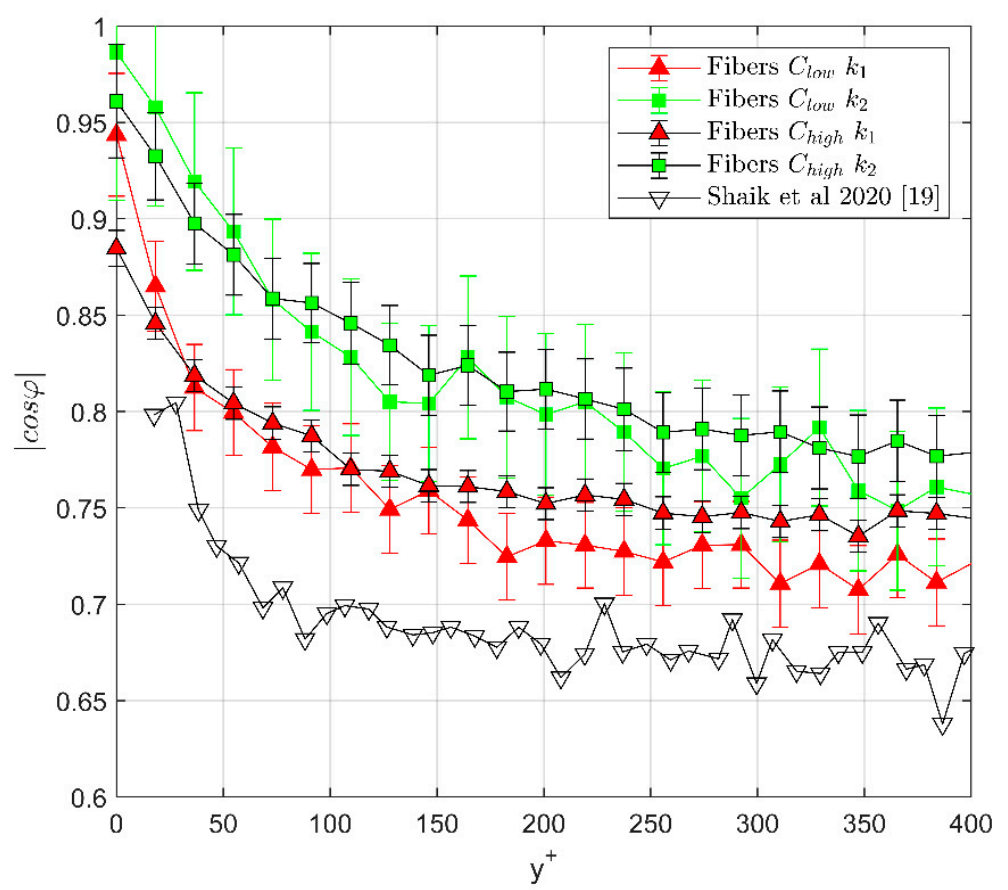

Figure 11. Average of cosine of fiber orientation angle $\varphi$, compared to experimental data from Shaik et al. (2020) [19]. Error bars indicate a 95\% confidence interval based on Student's t-distribution.

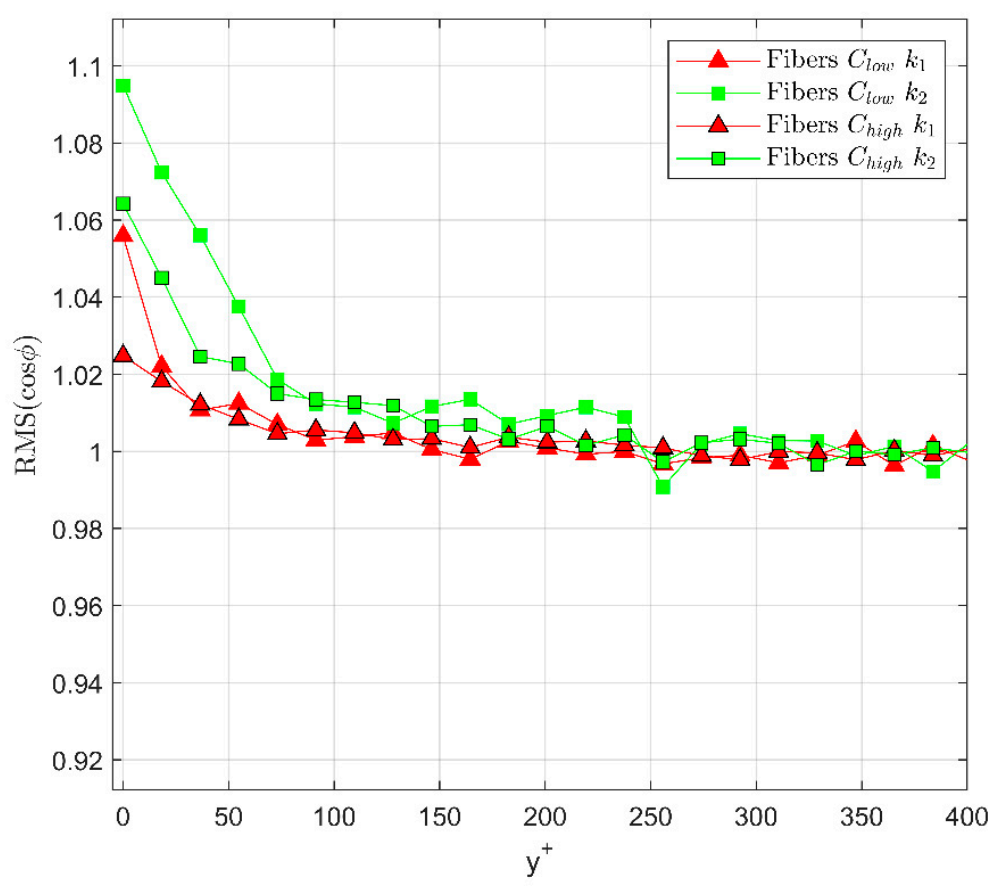

Figure 12. Root mean square (RMS) of fiber orientation angle fluctuations normalized to centerline value. 

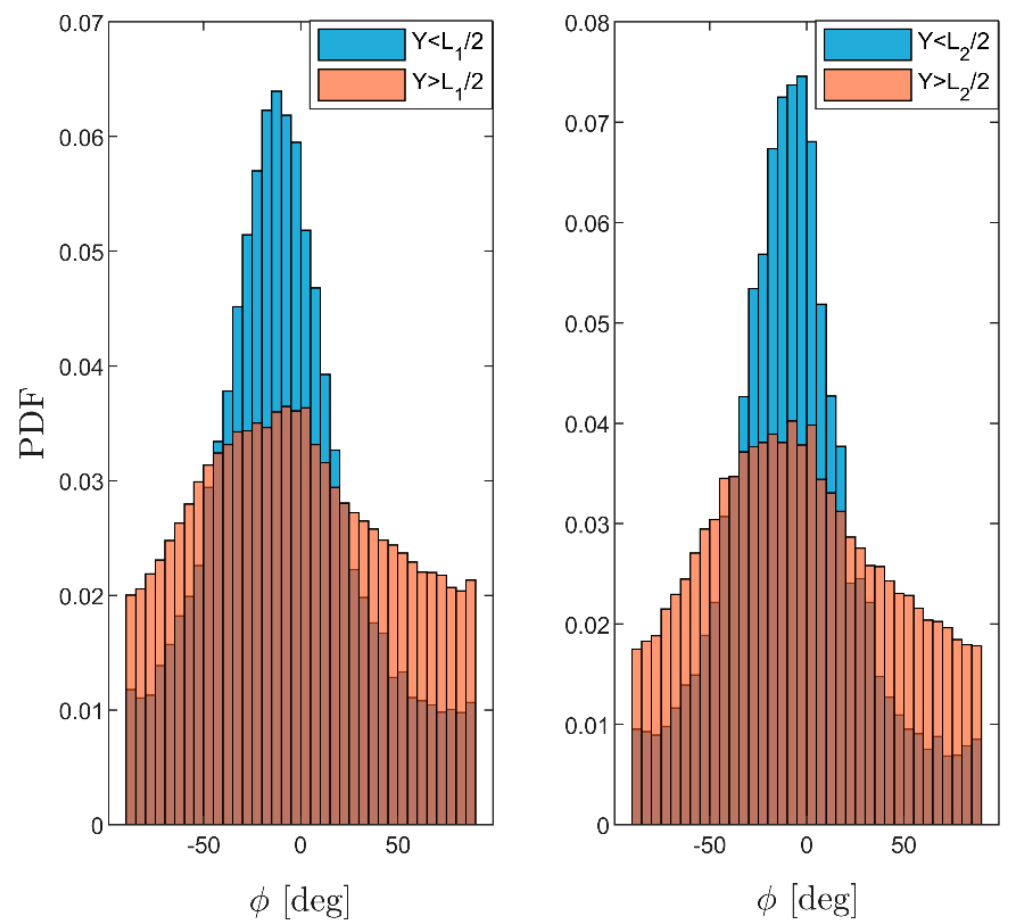

Figure 13. Estimate of probability density function of fiber orientation angle $\varphi$ conditioned to fiber distance from the wall. Low aspect ratio $k_{1}$ fibers (left), high aspect ratio $k_{2}$ fibers (right).

\section{Conclusions}

In this work a turbulent channel flow laden with elongated, fiber-like particles was experimentally investigated. Fibers with two different aspect ratios and two particle volume fractions were analyzed by means of optical methodologies. An image processing technique was implemented and set out to obtain separate carrier flow velocity data and fiber distribution and orientation information. Collected data sheds light on the role played by fiber aspect ratio on the dynamic coupling between dispersed and carrier phases. Turbulence modulation effects are reported in the near-wall region, with higher aspect ratio fibers associated with a larger turbulence increase in both horizontal and vertical velocity components of carrier flow. Higher-order statistical moments inspection shows an increase in the absolute value in the case of fiber-laden cases with respect to the clear-water reference case, in particular in the channel region far from the wall. An increase in the particle volume fraction is reported to be associated with higher turbulent fluctuations of the carrier flow. As regards the effect of the turbulent flow on a particles' behavior, preferential accumulation of fibers is reported in the wall proximity, up to the logarithmic layer. Within this region, fibers display different behavior according to their aspect ratio. In particular, fibers with lower aspect ratio reportedly tend to accumulate at a distance equal to their length from the wall before leveling at the centerline, whereas higher aspect ratio fibers exhibit an almost linear increase throughout the near-wall area. It is reasonable to assume that a crucial role in this mechanism is played by the relationship between particle length and the flow scales of the coherent structures of the turbulent boundary layer.

Fiber orientation data show that elongated particles are most likely to align to the horizontal axis $\mathrm{X}$ when they are in the wall proximity, in particular the ones featuring a higher aspect ratio. High levels of orientation angle fluctuations of particles are associated with carrier flow turbulence enhancement, suggesting that in multiphase flows laden with elongated particles, the primary mechanism of turbulence modulation is enhanced wake dynamics and induced vortex shedding by fiber motion. The analysis of probability of the orientation angle points out that, when scaled according to the fibers' length, conditional PDFs collapse to the same curves regardless of the particles' aspect ratio. 
Author Contributions: Conceptualization, A.C. and F.A.P.; methodology, A.C., F.A.P. and F.D.F.; software, A.C.; validation, A.C.; formal analysis, A.C.; investigation, A.C.; resources, F.A.P. and F.D.F.; data curation, A.C.; writing-original draft preparation, A.C.; project administration, F.A.P.; funding acquisition, F.D.F. and F.A.P. All authors have read and agreed to the published version of the manuscript.

Funding: This work was funded by the Italian Ministry of Research, PRIN project ACE, grant number 2017RSH3JY.

Institutional Review Board Statement: Not applicable.

Informed Consent Statement: Not applicable.

Data Availability Statement: Not applicable.

Conflicts of Interest: The authors declare no conflict of interest. The funders had no role in the design of the study; in the collection, analyses, or interpretation of data; in the writing of the manuscript, or in the decision to publish the results.

\section{References}

1. Crowe, C.T.; Gore, R.A.; Troutt, T.R. Particle dispersion by coherent structures in a free shear flow. Part Sci. Tech. J. 1985, 3, 149-158. [CrossRef]

2. Eaton, J.K.; Fessler, J.R. Preferential Concentration of particles by turbulence. Int. J. Multiph. Flow 1994, 20, 169-209. [CrossRef]

3. Balachandar, S.; Eaton, J.K. Turbulent Dispersed Multiphase Flow. Annu. Rev. Fluid Mech. 2010, 42, 111-133. [CrossRef]

4. Parthasarathy, R.N.; Faeth, G.M. Turbulence modulation in homogeneous dilute particle-laden flows. J. Fluid Mech. 1990, 220, 485-514. [CrossRef]

5. Kussin, J.; Sommerfeld, M. Experimental studies on particle behaviour and turbulence modification in horizontal channel flow with different wall roughness. Exp. Fluids 2002, 33, 143-159. [CrossRef]

6. Paris, A.D.; Eaton, J.K. Turbulence Attenuation in a Particle-Laden Channel Flow; Stanford University: Stanford, CA, USA, 2001.

7. Capone, A.; Romano, G.P.; Soldati, A. Experimental investigation on interactions among fluid and rod-like particles in a turbulent pipe jet by means of Particle Image Velocimetry. Exp. Fluids 2014, 56, 1-15. [CrossRef]

8. Parsheh, M.; Brown, M.; Aidun, C. On the orientation of stiff fibres suspended in turbulent flow in a planar contraction. J. Fluid Mech. 2005, 545, 245-269. [CrossRef]

9. Van Hout, R. Time resolved PIV measurements of the interaction of polystyrene beads with near-wall coherent structures in a turbulent channel flow. Int. J. Multiph. Flow 2011, 37, 346-357. [CrossRef]

10. Righetti, M.; Romano, G.P. Particle-fluid interactions in a plane near-wall turbulent flow. J. Fluid Mech. 2004, 505, 93-121. [CrossRef]

11. Jeffery, G.B. The Motion of Ellipsoidal Particles Immersed in a Viscous Fluid. Proc. R. Soc. Lond. Ser. A 1922, 102, 161-179.

12. Voth Greg, A.; Soldati, A. Anisotropic particles in turbulence. Annu. Rev. Fluid Mech. 2017, 49, 249-276. [CrossRef]

13. Marchioli, C.; Fantoni, M.; Soldati, A. Orientation, distribution and deposition of elongated, inertial fibres in turbulent channel flow. Phys. Fluids. 2010, 22, 033301. [CrossRef]

14. Capone, A.; Romano, G.P. Interactions between fluid and fibers in a turbulent backward-facing step flow. Phys. Fluids 2015, 27, 053303. [CrossRef]

15. Sabban, L.; Cohen, A.; van Hout, R. Temporally resolved measurements of heavy, rigid fibre translation and rotation in nearly homogeneous isotropic turbulence. J. Fluid Mech. 2017, 814, 42-68. [CrossRef]

16. Eshghinejadfard, A.; Hosseini, S.A.; Thévenin, D. Fully-resolved prolate spheroids in turbulent channel flows: A lattice Boltzmann study. AIP Adv. 2017, 7, 095007. [CrossRef]

17. Zhao, L.; Challabotla, N.R.; Andersson, H.I.; Variano, E.A. Rotation of Nonspherical Particles in Turbulent Channel Flow. Phys. Rev. Lett. 2015, 115, 244501. [CrossRef] [PubMed]

18. Parsa, S.; Guasto, J.; Kishore, K.; Ouellette, N.; Gollub, J.; Voth, G. Rotation and alignment of rods in two dimensional chaotic flow. Phys. Fluids 2011, 23, 043302. [CrossRef]

19. Alipour, M.; De Paoli, M.; Ghaemi, S.; Soldati, A. Long non-axisymmetric fibres in turbulent channel flow. J. Fluid Mech. 2021, 916, A3. [CrossRef]

20. Shaik, S.; Kuperman, S.; Rinsky, V.; van Hout, R. Measurements of length effects on the dynamics of rigid fibers in a turbulent channel flow. Phys. Rev. Fluids 2020, 5, 114309. [CrossRef]

21. Ardekani, M.N.; Costa, P.; Breugem, W.P.; Picano, F.; Brandt, L. Drag reduction in turbulent channel flow laden with finite-size oblate spheroids. J. Fluid Mech. 2017, 816, 43-47. [CrossRef]

22. Zhu, C.; Yu, Z.; Shao, X. Interface-resolved direct numerical simulations of the interactions between neutrally buoyant spheroidal particles and turbulent channel flows. Phys. Fluids 2018, 30, 115103. [CrossRef]

23. Zhu, C.; Yu, Z.; Pan, D.; Shao, X. Interface-resolved direct numerical simulations of the interactions between spheroidal particles and upward vertical turbulent channel flows. J. Fluid Mech. 2020, 891. [CrossRef] 
24. Gore, R.A.; Crowe, C.T. Effect of particle size on modulating turbulent intensity. Int. J. Multiph. Flow 1989, 15, 279-285. [CrossRef]

25. Khalitov, D.A.; Longmire, E.K. Simultaneous two-phase PIV by two-parameter phase discrimination. Exp. Fluids 2002, 32, 252-268. [CrossRef]

26. Christian, P.; Westerweel, J.; Ooms, G. Turbulence statistics from optical whole-field measurements in particle-laden turbulence. Exp. Fluids 2006, 40, 347-363.

27. Paris, A. Measuring velocity gradients in a particle-laden channel flow. In Proceedings of the Third International Workshop on Particle Image Velocimetry PIV'99, Santa Barbara, CA, USA, 16-18 September 1999.

28. Tomohiko, T.; Eaton, J.K. Sub-Kolmogorov resolution partical image velocimetry measurements of particle-laden forced turbulence. J. Fluid Mech. 2010, 643, 177-206.

29. Tomohiko, T.; Eaton, J.K. Classification of turbulence modification by dispersed spheres using a novel dimensionless number. Phys. Rev. Lett. 2008, 101, 114502.

30. Ahmadi, F.; Ebrahimian, M.; Sanders, R.S.; Ghaemi, S. Particle image and tracking velocimetry of solid-liquid turbulence in a horizontal channel flow. Int. J. Multiph. Flow 2019, 112, 83-99. [CrossRef]

31. Parsheh, M.; Brown, M.; Aidun, C. Variation of fiber orientation in turbulent flow inside a planar contraction with different shapes. Int. J. Multiph Flow 2006, 32, 1354-1369. [CrossRef]

32. Krochak, P.; Olson, J.; Martinez, D. The orientation of semidilute rigid fiber suspensions in a linearly contracting channel flow. Phys. Fluids 2008, 20, 073303. [CrossRef]

33. Holm, R.; Soederberg, D. Shear influence on fibre orientation: Dilute suspension in the near wall region. Rheol. Acta 2007, 46, 721-729. [CrossRef]

34. Kasagi, N.; Matsunaga, A. Three-dimensional particle-tracking velocimetry measurement of turbulence statistics and energy budget in a backward-facing step flow. Int. J. Heat Fluid Flow 1995, 16, 477-485. [CrossRef]

35. Kiger, K.T.; Pan, C. PIV Technique for the simultaneous Measurement of Dilute Two-Phase Flows. J. Fluid Eng. Trans. ASME 2000, 122, 811-817. [CrossRef]

36. Elghobashi, L. On predicting particle-laden turbulent flows. App. Sci. Resh. 1994, 52, 309-329. [CrossRef]

37. Moses, K.B.; Advani, S.G.; Reinhardt, A. Investigation of fiber motion near solid boundaries in simple shear flow. Rheol. Acta 2001, 40, 296-306. [CrossRef]

38. Do-Quang, M.; Amberg, G.; Brethouwer, G.; Johansson, A.V. Simulation of finite-size fibers in turbulent channel flows. Phys. Rev. E 2014, 89, 013006. [CrossRef]

39. Krochak, P.J.; Olson, J.A.; Martinez, D.M. Near-wall estimates of the concentration and orientation distribution of a semi-dilute rigid fibre suspension in Poiseuille flow. J. Fluid Mech. 2010, 653, 431-462. [CrossRef] 